\title{
Coagulation pathways and diabetic retinopathy: abnormal modulation in a selected group of insulin dependent diabetic patients
}

\author{
Cristiano Giusti, Riccardo Schiaffini, Claudia Brufani, Antonio Pantaleo, \\ Enzo Maria Vingolo, Patrizia Gargiulo
}

\begin{abstract}
Aims-To investigate whether diabetic retinopathy (DR), already associated with microvascular alterations, ischaemia, and endothelial dysfunction, was also characterised by abnormal modulation of coagulation pathways.

Methods-Plasma samples, collected from 67 type 1 diabetics comparable for age, duration of disease (DD), and metabolic control (MC), were processed for prothrombin degradation products $(F 1+2)$ and factor VII coagulant activity (FVII:c). 50 normal subjects served as a control group. The ETDRS-Airlie House Classification of DR was used.

Results-A significant correlation between FVII:c and F1+2 plasma concentrations was observed $(p<0.05)$. FVII:c (p $<0.005)$ and $F 1+2(p<0.0001)$ levels were higher in diabetics than in controls, especially in patients with proliferative $D R$ (FVII:c p <0.0001; F1+2 p<0.005). However, cases without retinal lesions and healthy subjects did not differ significantly (FVII:c and F1+2 p >0.05).

Conclusions-These findings pointed out the presence of a hypercoagulable state associated with endothelial dysfunction in patients with insulin dependent diabetes mellitus (IDDM), demonstrated both by increased FVII:c and F1+2 plasma levels. Moreover, the observation of different DR related degrees of procoagulant activity, despite comparable DD and MC, strengthens the hypothesis of multiple risk factors in the pathogenesis of DR. (Br F Ophthalmol 2000;84:591-595)
\end{abstract}

Chair of

Ophthalmology,

University "La

Sapienza", Rome, Italy

C Giusti

E M Vingolo

Institute of Internal

Medicine II

R Schiaffini

C Brufani

A Pantaleo

P Gargiulo

Correspondence to: Cristiano Giusti, MD, Via Cassia 1280 (Pal B1, int 10), I-00189 Rome, Italy

crigiust@tin.it

Accepted for publication 28 January 2000

Diabetic retinopathy (DR) is the major cause of impaired vision in the Western world and of new cases of blindness for people between the ages of 20 and 64 years. ${ }^{1}$ However, despite the growing concern about this disease, its natural history and aetiopathogenesis are still not completely understood: several risk factors have been identified ${ }^{2-16}$ and much speculation surrounds the pathophysiology of diabetic microvascular lesions but, at this time, no data provide an adequate explanation for the serious and rapid involvement of the retinal microcirculation that often occur early in the disease despite a good metabolic control. The Diabetes Control and Complications Trial Research Group pointed out that intensive insulin therapy resulted in a $76 \%$ reduction in progression of retinopathy during a 9 year period in patients with insulin dependent diabetes mellitus (IDDM) ${ }^{17}$ : however, a sight threatening worsening is often observed when this intensive treatment is initiated in patients with long standing poor glycaemic control, particularly if retinopathy is at or past the moderate non-proliferative stage. ${ }^{18}$ Moreover, the severity of DR is probably influenced also by familial factors. ${ }^{19}$

Experimental evidence suggests that hyperglycaemia triggers a cascade of biochemical events that lead to vascular dysfunction and early structural changes of blood vessel walls. ${ }^{20}$ Several observations that vascular damage and endothelial dysfunction occur early in the course of diabetic microangiopathy have been reported in the literature: increased endothelin-1 (ET-1) levels ${ }^{21}$; increased plasma concentrations of tissue plasminogen activator (tPA) inhibitor, ${ }^{23}$ von Willebrandantihaemophilic factor $\mathrm{A},{ }^{24}{ }^{25}$ fibrinogen and activated factor VII (FVII:c) ${ }^{26}$; decreased concentrations of endothelium derived relaxing factor, prostacyclin, ${ }^{25}$ and $\mathrm{tPA}^{23}$; and reduced fibrinolytic potential of vascular endothelia. ${ }^{27} 28$ The net effect of all these changes is to convert the endothelium from a thromboresistant to a thrombogenic surface ${ }^{29} 30$ and, consequently, impairment of coagulation and of anticoagulant pathways. ${ }^{31-33}$

In the light of all these findings, the aim of the present study was to determine, in a well characterised group of patients affected by insulin dependent diabetes mellitus (IDDM), whether DR was associated with abnormally modulated haemostasis and to demonstrate whether or not a significant correlation existed between the thrombotic tendency, as measured by FVII:c and prothrombin degradation fragments $(\mathrm{F} 1+2)$ plasma concentrations, and metabolic control.

\section{Subjects and methods}

STUDY POPULATION

Sixty seven IDDM patients (22 males, 45 females; age range 15-68 years), classified according to the National Diabetes Data Group criteria, ${ }^{34}$ regularly attending the Center for the Study of Diabetes (Institute of Internal Medicine II, University of Rome "La Sapienza") from 1989 to the end of 1996 were enrolled. Selection was made by the same diabetologist on the basis of the following inclusion criteria: type 1 IDDM, as evidenced by deficient $\mathrm{C}$ peptide secretion, with a 
duration of the disease longer than 5 years. Poor glycaemic control $\left(\mathrm{HbA}_{1 c}>7 \%\right)$, borderline hypertension $(>140 / 90)$, hypertriglyceridaemia $(>1.9 \mathrm{mmol} / \mathrm{l})$, or hypercholesterolaemia $(>5.6 \mathrm{mmol} / \mathrm{l})$, detected on three different occasions, as well as peripheral obliterating arteriopathies or other macrovascular diseases were exclusion criteria. Fifty healthy, nondiabetic subjects, aged between 20 and 50 years, recruited from hospital staff, served as a control group.

Medical history, physical examination, electrocardiography, and Doppler analysis of extracranial and intracranial vessels were used to investigate the presence of macroangiopathy and venous or arterial thrombosis (for example, cerebral or coronary).

Nephropathy was diagnosed by the presence of microalbuminuria (30-300 mg/day: Albustix-Ames, Miles, Elkhart, IN, USA) or macroalbuminuria (>300 mg/day: Labstix, Bayer Inc, Bridgend) in at least three samples of early morning urine collected in a 6 month period in the absence of infections, other renal diseases, or heart failure. ${ }^{35}$ Blood pressure and serum creatinine concentration were determined in order to evaluate renal function.

The ophthalmological examination included: best corrected ETDRS visual acuity, applanation tonometry, and biomicroscopic evaluation of anterior segment. DR was assessed according to the ETDRS-Airlie House Classification ${ }^{36}$ : binocular indirect ophthalmoscopy, retinal biomicroscopy using high positive power precorneal lenses (Super Field and/or +78D Volk), and colour fundus stereophotographs (and fluorescein angiography, if necessary) were performed through dilated pupils.

All patients were treated with subcutaneous human insulin (regular and long acting) only. Both diabetics and controls were non-smokers and had not taken drugs which could affect haemostasis for at least 4 weeks ( 6 months in the case of oral contraceptives) before the study.

COLLECTION AND PROCESSING OF BLOOD

SAMPLES

Fasting blood samples were collected using 19 gauge needles and polypropylene syringes preloaded with the appropriate solutions. All samples were drawn between 830 and 930 $\mathrm{am}$, in a quiet room, following a minimum of 30 minutes of rest; blood drawing was performed without arm compression to avoid even minimal venous stasis and prevent platelet activation.

Glycosylated haemoglobin $\left(\mathrm{HbA}_{1 \mathrm{c}}\right)$ was determined spectrophotometrically using reagents from Bio-Rad (Richmond, CA, USA) on $3 \mathrm{ml}$ of blood drawn into evacuated siliconised tubes containing EDTA. The non-diabetic range used in our laboratory is $4.0-6.0 \%$. To determine serum glucose and serum lipids (total cholesterol, triglycerides, high density lipoprotein cholesterol), blood was drawn into evacuated siliconised tubes and measurements made by autoanalyser (Boehringer Mannheim, Germany) using enzymatic methods.
In order to determine factor VII antigen (FVII:Ag), factor VII coagulant activity (FVII:c), and prothrombin degradation fragments $(\mathrm{F} 1+2)$, blood was collected directly into evacuated siliconised tubes containing $0.129 \mathrm{M}$ buffered trisodium citrate, one part to nine parts blood, volume for volume. Samples, identified by the initials of the subjects and date of sampling only in order to leave the analysts masked to the DR diagnosis of each patient, were immediately shipped to the laboratory and processed within 1 hour of collection. Plasma was obtained by centrifugation at $2000 \mathrm{~g}$ at $4^{\circ} \mathrm{C}$ for 60 minutes, separated into small aliquots, immediately stored in plastic tubes, and frozen at $-80^{\circ} \mathrm{C}$ until assayed.

FVII:Ag was measured with a commercial immunoenzymatic ELISA method (Diagnostica Stago, France $)^{37}$ and results expressed as a percentage of those given by standard plasma (\% standard).

FVII:c was determined by a clotting time assay using factor VII deficient plasma as reagent (Boehringer Mannheim, Germany), according to the method described by Poggio. ${ }^{38}$

The prothrombin degradation products $(\mathrm{F} 1+2)$ assay measures the accumulation of intermediate molecules from the conversion of prothrombin to thrombin in blood. This was performed using double antibody RIA employing labelled and unlabelled F1+2 (Behring Werke AG, Marburg, Germany). ${ }^{39}$

All assays were duplicate and results for each patient remained masked to the ophthalmologists until data processing was completed.

Informed consent was obtained from all subjects after full explanation of the nature of the study. This investigation was approved by the bioethics committee of the University of Rome "La Sapienza" and followed the tenets of the Declaration of Helsinki.

\section{STATISTICAL ANALYSES}

Analyses were performed by a statistician provided with a list of the patients' laboratory values but masked to the clinical diagnosis of diabetics and the identity of healthy control subjects. Upon completion of analyses, masking was broken, and assay results were matched to the clinical diagnoses. Data were expressed as mean (SD) and were normally distributed, except for age and $\mathrm{HbA}_{1 \mathrm{c}}$. Statistical analyses were performed by Mann-Whitney $U$ test for independent groups for the significance of differences. Bonferroni's correction was applied to control for the increase of type 1 error probability due to multiple comparison. A p value of less than 0.05 was considered statistically significant.

\section{Results}

CLINICAL AND METABOLIC RESULTS

Clinical, biochemical, and haemodynamic variables were comparable in diabetics and healthy controls (Table 1): no statistically significant differences were found between both groups of subjects, with the exception of $\mathrm{HbA}_{1 \mathrm{c}}$ and creatinine.

DR was found in $45(67.1 \%)$ cases, associated with a higher mean age and a longer 
Table 1 Clinical, biochemical, and haemostatic findings of diabetic patients and healthy controls ( $n=$ mean (SD); $N s=p$ value not significant)

\begin{tabular}{llll}
\hline & Diabetics $(n=67)$ & Controls $(n=50)$ & $p$ Value \\
\hline Age (years) & $39.3(15.2)$ & $37.4(6.6)$ & $\mathrm{Ns}$ \\
Sex (M, F) & 22,45 & 19,31 & - \\
Duration of diabetes (years) & $16.9(8.43)$ & - & - \\
Body mass index (kg/m $\left.{ }^{2}\right)$ & $24.1(3.54)$ & $22.9(2.04)$ & $\mathrm{Ns}$ \\
Systolic blood pressure (mm Hg) & $127.0(15.4)$ & $127.8(11.26)$ & $\mathrm{Ns}$ \\
Diastolic blood pressure (mm Hg) & $76.6(8.68)$ & $72.3(8.28)$ & $\mathrm{Ns}$ \\
HbA $_{\text {lc }}(\%)$ & $6.17(0.90)$ & $4.54(0.64)$ & $<0.0001$ \\
Nephropathy: $_{\quad}$ microalbuminuria (patients) & $16(23.8 \%)$ & - & - \\
$\quad$ macroalbuminuria (patients) & $9(13.4 \%)$ & - & - \\
Total cholesterol (mg/dl) & $188.5(13.3)$ & $189.6(22.7)$ & $\mathrm{Ns}$ \\
HDL cholesterol (mg/dl) & $49.2(5.2)$ & $50.4(3.5)$ & $\mathrm{Ns}$ \\
Triglycerides (mg/dl) & $143.1(34.1)$ & $144.6(28.2)$ & $\mathrm{Ns}$ \\
Creatinine (mmol/l) & $83.98(32.70)$ & $71.60(13.26)$ & $<0.005$ \\
FVII:Ag (\% standard) & $110.4(32.1)$ & $102.9(29.8)$ & $<0.05$ \\
FVII:c (U/dl) & $99.2(17.5)$ & $88.6(15.8)$ & $<0.005$ \\
F1+2 ( $\mu$ g/l) & $1.12(0.46)$ & $0.78(0.24)$ & $<0.0001$ \\
\hline
\end{tabular}

duration of diabetes compared to those without retinal lesions $(\mathrm{p}<0.05)$ : background $(\mathrm{n}=$ 21 ), non-proliferative $(\mathrm{n}=15)$, and proliferative retinopathy $(n=9)$ were observed (Table 2). Other risk factors for severity of $D R$ - for example, sex and $\mathrm{HbA}_{1 \mathrm{c}}$ values, were statistically insignificant in this study $(\mathrm{p}>0.05)$.

Microalbuminuria was detected in 16 diabetics $(23.8 \%)$, mostly associated with proliferative DR $(n=9)$ but also with background ( $n$ $=2)$ or non-proliferative retinopathy $(n=5)$. Macroalbuminuria, on the contrary, was observed only in those diabetics affected by proliferative DR ( $\mathrm{n}=9 ; 13.4 \%$ ) (Tables 1 and 2).

\section{COAGULATION STATUS}

Clinical, metabolic, and haemocoagulative data of cases and healthy controls are shown in Tables 1 and 2. In diabetics, a significant correlation between FVII:c and F1+2 plasma concentrations was observed $(R=0.378, p$ $<0.05)$. FVII:Ag ( $\mathrm{p}<0.05)$, FVII:c $(\mathrm{p}<0.005)$ and $\mathrm{F} 1+2(\mathrm{p}<0.0001)$ levels were higher in diabetics than in controls (Table 1), especially in patients with proliferative DR (FVII:Ag: $\mathrm{p}<$ 0.005; FVII:c: $\mathrm{p}<0.0001$; F1+2: $\mathrm{p}<0.005)$. Cases without retinal lesions and healthy controls did not differ significantly (FVII:Ag, FVII:c and F1+2: $p>0.05)$.

\section{Discussion}

According to epidemiological studies, about $89 \%$ of IDDM patients have a clinically evident retinopathy 10 years after onset of the disease. Ten years later, this percentage increases to $99.0 \%$ and the prevalence of the proliferative form is about $53 \% .{ }^{7}$ In our group of patients, characterised by a mean duration of the disease of about 17 years associated with a good metabolic control $\left(\mathrm{HbA}_{1 \mathrm{c}}=6.17 \%\right.$ $(0.90 \%)$ (Table 1$)$, the overall prevalence of DR was lower $(67.1 \%)$ with a percentage of proliferative retinopathy of $20 \%$. These data strengthens the hypothesis of a multifactorial pathogenesis of diabetic vasculopathy in which the glycometabolic factor plays an important but not exclusive part. ${ }^{1-19}$

In the light of the different pathogenetic hypotheses and the several risk factors that have been already reported in the literature, this study was designed to determine whether DR was associated with abnormally modulated haemostasis, as a result, and expression of endothelial damage. In fact, there are many reports that extensively document the presence of a remarkable endothelium related dysfunction of the coagulant and anticoagulant pathways in diabetics, but it is not yet clear if this condition is due to hyperglycaemia only. ${ }^{20} 23-28$ 31-33 Abnormal coagulation is manifested by enhanced prothrombin conversion to thrombin-as demonstrated by increased $\mathrm{F} 1+2$ plasma concentrations ${ }^{40}$ - and downregulation of the anticoagulant pathway, caused by reduced antithrombin III (ATIII) activity and thrombomodulin endothelial receptors. ${ }^{41}$

Increased FVII:c plasma levels have also been observed in diabetics, ${ }^{42} 43$ especially in association with chronic vascular complications. ${ }^{44}{ }^{45}$ Moreover, it has been demonstrated that high FVII coagulant activity, associated with increased $\mathrm{FXa}$ production but low thrombin and F1+2 levels, characterises the prethrombotic condition, ${ }^{46}$ whereas increased F1+2 and FVII:c plasma concentrations are typical features of the hypercoagulable state. ${ }^{46}{ }^{47}$

Table 2 Clinical, laboratory, and haemocoagulative data in diabetics (in relation to diabetic retinopathy: BDR = background DR; NPDR = non-proliferative DR;PDR = proliferative DR) and in healthy controls $(n=$ mean $(S D)$

\begin{tabular}{|c|c|c|c|c|c|}
\hline & No $D R(n=22)$ & $B D R(n=21)$ & $\operatorname{NPDR}(n=15)$ & $\operatorname{PDR}(n=9)$ & Controls $(n=50)$ \\
\hline $\operatorname{Sex}(M / F)$ & $9 / 13$ & $5 / 15$ & $2 / 5$ & $6 / 12$ & $19 / 31$ \\
\hline Age range (years) & $16-55$ & $15-62$ & $16-65$ & $24-70$ & $20-68$ \\
\hline Mean age (years) & $36.4(10.5)$ & $41.2(17.3)$ & $42.10(12.3)$ & $43.6(13.1)$ & $38.6(12.2)$ \\
\hline Duration of diabetes (years) & $11.4(6.9)$ & $17.8(5.7)$ & $18.0(1.2)$ & $18.1(7.2)$ & - \\
\hline $\mathrm{HbA}_{1 \mathrm{c}}(\%)$ & $6.1(1.0)$ & $6.2(1.1)$ & $6.3(1.2)$ & $6.6(1.0)$ & - \\
\hline Microalbuminuria (patients) & - & 2 & 5 & 9 & - \\
\hline Macroalbuminuria (patients) & - & - & - & 9 & - \\
\hline Total cholesterol $(\mathrm{mg} / \mathrm{dl})$ & $180.9(31.0)$ & $187.5(46.0)$ & $192.1(31.1)$ & $204.2(49.4)$ & $189.6(22.7)$ \\
\hline HDL cholesterol (mg/dl) & $51.4(9.8)$ & $47.8(6.7)$ & $47.2(5.2)$ & $46.4(8.4)$ & $50.4(3.5)$ \\
\hline Triglycerides (mg/dl) & $118.8(52.6)$ & $131.5(46.8)$ & $140.1(31.3)$ & $165.2(34.5)$ & $144.6(28.2)$ \\
\hline FVII:Ag (\% standard) & $98.6(33.1)$ & $108.8(30.9)$ & $117.6(22.8)$ & $131.6(21.7)^{\star}$ & $102.9(29.8)$ \\
\hline FVII:c (U/dl) & $79.7(18.4)$ & $92.8(16.9)$ & $105.3(10.4)$ & $126.0(34.3)^{\star \star}$ & $88.6(15.8)$ \\
\hline $\mathrm{F} 1+2(\mu \mathrm{g} / \mathrm{l})$ & $0.82(0.46)$ & $1.08(0.58)$ & $1.07(0.43)$ & $1.41(0.61)^{\star \star \star}$ & $0.78(0.24)$ \\
\hline
\end{tabular}

$\star$ PDR $v$ No DR, PDR $v$ BDR, PDR $v$ controls: $\mathrm{p}<0.005$; PDR $v$ NPDR: $\mathrm{p}<0.05$

No DR $v$ controls: $\mathrm{p}>0.05$ (not significant).

$\star \star$ PDR $v$ No DR, PDR $v$ BDR, PDR $v$ controls: $\mathrm{p}<0.0001$; PDR $v$ NPDR: $\mathrm{p}<0.05$

No DR $v$ controls: $\mathrm{p}>0.05$ (not significant)

$\star \star \star$ PDR $v$ No DR, PDR $v$ controls: $\mathrm{p}<0.005$; PDR $v$ BDR, PDR $v$ NPDR: $\mathrm{p}<0.05$

No DR $v$ controls: $\mathrm{p}>0.05$ (not significant). 
In this study, in order to minimise metabolic interference with our data, all diabetics were under a good metabolic control $\left(\mathrm{HbA}_{1 \mathrm{c}}<7 \%\right)$. No thrombotic events in large vessels were observed, probably due to the chronic nature of the endothelial dysfunction.

Diabetics without retinopathy had significantly lower FVII:Ag ( $\mathrm{p}<0.005)$, FVII:c (p $<0.0001)$, and $F 1+2(\mathrm{p}<0.005)$ plasma levels than those with proliferative DR, but comparable levels with healthy controls (FVII:Ag, FVII:c, and F1+2: p >0.05) (Table 2). These data suggest that in patients without retinal lesions, despite the hyperglycaemic condition, the vascular endothelium preserves its physiological thromboresistance. However, they indicate also that in cases of proliferative DR a hypercoagulable state is present: in particular, the high FVII:Ag plasma concentrations might predispose the extrinsic pathway to a hyperactivation and, consequently, the retinal microcirculation to a much more severe involvement, as a result either of endothelial dysfunction ${ }^{48}$ or of gradually overwhelmed anticoagulant systems. ${ }^{47}$ In conclusion, these results confirm that a stable and good metabolic control $\left(\mathrm{HbA}_{1 \mathrm{c}}<7 \%\right)$ reduces the incidence of $\mathrm{DR}^{34}$; point out that a hypercoagulable state is present in type 1 diabetics with retinopathy; support the main part played by the endothelial dysfunction as demonstrated both by activation of the extrinsic haemostatic pathway and by increased concentrations of prothrombin degradation fragments. Particularly, the association observed between increased FVII:Ag, FVII:c, and F1+2 plasma levels supports the opinion that the endothelium in type 1 diabetics with proliferative DR, is functionally converted from a thromboresistant to a thrombogenic surface. Moreover, the findings of different DR related degrees of procoagulant activity, despite comparable duration of the disease and metabolic control, strengthens the hypothesis of multiple risk factors in the pathogenesis of DR.

Further investigations on larger diabetic populations are necessary to clarify and support this issue. If these data were confirmed by other authors, it would be possible not only to reach a better understanding of the pathogenesis of DR but also to prevent its onset and/or progression, helping primary care physicians and ophthalmologists in detecting patients at risk when they can still be treated effectively with conservative methods (for example, drugs with antithrombotic, profibrinolytic, and cytoprotective effects able to restore the endothelial function ${ }^{49}$ ) and before the need for laser treatments that always destroy the retinal anatomy.

This work was presented in part at the annual meeting of the Association for Research in Vision and Ophthalmology (ARVO), Fort Lauderdale, FL, USA, 10-15 May 1998.

1 Moss SE, Klein R, Klein BEK. The 14-year incidence of vision loss in a diabetic population. Ophthalmology 1998;105:998-1003.

2 Klein R, Klein BEK, Moss SE. Retinopathy in young-onset diabetic patients. Diabet Care 1985;8:311-5.

3 Klein R, Klein BEK, Moss SE, et al. Glycosylated hemoglobin predicts the incidence and progression of diabetic retinopathy. FAMA 1988;260:2864-71.
4 Diabetes Control and Complication Trial. The relationship of glycemic exposure $\left(\mathrm{HbA}_{1 \mathrm{c}}\right)$ to the risk of development and progression of retinopathy in the diabetes control and complication trial. Diabetes 1995;44:968-83.

5 Klein R, Klein BEK, Moss SE, et al. Is blood pressure a predictor of the incidence or progression of diabetic retinopathy? Arch Intern Med 1989;149:2427-32.

6 Klein R, Klein BEK, Moss SE, et al. The relationship of hyperglycemia to long-term incidence and progression of diabetic retinopathy. Arch Intern Med 1994;154:2169-78.

7 Klein R, Klein BEK, Moss SE, et al. The Wisconsin Epidemiologic Study of Diabetic Retinopathy. XIV. Ten-year incidence and progression of diabetic retinopathy. Arch Ophthalmol 1994;112:1217-28.

8 Rand LI, Krolewski AS, Aiello LM, et al. Multiple factors in the prediction of risk of proliferative diabetic retinopathy. $N$ Engl f Med 1985;313:1433-8.

9 Klein R, Klein BEK, Moss SE. The Wisconsin Epidemiologic Study of Diabetic Retinopathy. XVI. The relationship of C-peptide to the incidence and progression of diabetic retinopathy. Diabetes 1995;44:796-801.

10 Dornan TL, Ting A, McPherson CK. Genetic susceptibility to the development of retinopathy in insulin-dependent diabetics. Diabetes 1982;31:226-31.

11 Baker RS, Rand LI, Krolewski AS, et al. Influence of HLA-DR phenotype and myopia on the risk of nonproliferative and proliferative diabetic retinopathy. Am f Ophthalmol 1986;102:693-700.

12 Klein R, Klein BEK, Davis MD. Is cigarette smoking associated with diabetic retinopathy? Am $\mathcal{F}$ Epidemiol 1983;118: $228-38$

13 Moss SE, Klein R, Klein BEK. Ocular factors in the incidence and progression of diabetic retinopathy. Ophthalmology 1994;101:77-83.

14 Giusti C, Pannarale L, Pazzaglia S, et al. Incidence of diabetic retinopathy in myopic eye. (ARVO abstract) Invest Ophthalmol Vis Sci 1996;37(4 suppl):S958.

15 Klein R. Barriers to prevention of vision loss caused by diabetic retinopathy. Arch Ophthalmol 1997;115:1073-5.

16 Baker RS, Watkins NL, Wilson MR, et al. Demographic and clinical characteristics of patients with diabetes presenting to an urban public hospital ophthalmology clinic. Ophthalto an urban public hospital
mology 1998;105:1373-9.

17 The Diabetes Control and Complications Trial Research Group. Progression of retinopathy with intensive versus conventional treatment in the Diabetes Control and Complications Trial. Ophthalmology 1995;102:647-61.

18 The Diabetes Control and Complications Trial Research Group. Early worsening of diabetic retinopathy in the Diabetes Control and Complications Trial. Arch Ophthalmol 1998;116:874-86.

19 The Diabetes Control and Complications Trial Research Group. Clustering of long-term complications in families with diabetes in the diabetes control and complications trial. Diabetes 1997;46:1829-39.

20 Lorenzi M, Cagliero E. Pathobiology of endothelial and other vascular cells in diabetes mellitus: call for data. Diabetes 1991;40:653-9.

21 Laurenti O, Vingolo EM, Desideri GB, et al. Increased levels of plasma endothelin-1 in non-insulin dependent diabetic patients with retinopathy but without other diabetes-related organ damage. Exp Clin Endocrinol Diabetes 1997;105:40-2.

22 Letizia C, Iannaccone A, Cerci S, et al. Circulating endothelin-1 in non-insulin-dependent diabetic patients with retinopathy. Horm Metab Res 1997;29:247-51.

23 Walmsley D, Hampton KK, Grant PF. Contrasting fibrinolytic responses in type 1 (insulin-dependent) and Type 2 (non-insulin-dependent) diabetes. Diabetic Med 1991;8: 954-9.

24 Pandolfi $M$, Almèr LO, Holmberg L. Increased von Willebrand-antihaemophilic factor A in diabetic retinopathy. Acta Ophthalmol Scand 1974;52:823-8.

25 Brenner BM, Troy JL, Ballermann BJ. Endotheliumdependent vascular responses: mediator and mechanism. $\mathcal{F}$ Clin Invest 1989;84:1373-8.

26 Fuller JH, Keen H, Jarret RJ. Haemostatic variables associated with diabetes and its complications. BMF 1979;2:9646.

27 Garcia Frade LJ, De la Calle H, Torado MC, et al. Hypofibrinolysis associated with vasculopathy in non-insulindependent diabetes mellitus. Thromb Res 1990;59:51-9.

28 Mansfield MW, Grant PJ. Fibrinolysis and diabetic retinopathy in NIDDM. Diabetes Care 1995;18:1577-81.

retinopathy in NIDDM. Diabetes Care 1995; $18: 1577-81$.
Haefliger IO, Meyer P, Flammer J, et al. The vascular Haefliger IO, Meyer P, Flammer J, et al. The vascular
endothelium as a regulator of the ocular circulation: a new concept in ophthalmology? Surv Ophthalmol 1994;39:12332 .

30 Kohner EM, Patel V, Rassman SMB. Role of blood flow and impaired autoregulation in the pathogenesis of diabetic retinopathy. Diabetes 1995;44:603-7.

31 Ceriello A. Coagulation activation in diabetes mellitus: the role of hyperglycemia and therapeutic prospects. Diabetologia 1993;36:1119-25.

32 Colmann RW, Hirsh J, Marder VJ, et al. Retinal microangiopathy, hemostasis and thrombosis: basic principles and clinical practice. Philadelphia: Lippincott, 1987.

33 Matsuda T, Morishita E, Jokaij H, et al. Mechanism on disorders of coagulation and fibrinolysis in diabetes. Diabetes 1996;45(3 suppl):S109-10.

34 National Diabetes Data Group. Classification of diabetes mellitus and other categories of glucose intolerance. Diabetes 1979;28:1039-57. 
35 Mogensen CE, Schmitz O. The diabetic kidney: from hyperfiltration and microalbuminuria to end-stage renal failure. Med Clin N Am 1988;72:1465.

36 Diabetic Retinopathy Study Research Group. Report 7. A modification of the Airlie House classification of diabetic retinopathy. Invest Ophthalmol Vis Sci 1981;21:210-26.

37 Boyer C, Wolf M, Rotschild C, et al. An enzyme immunoassay (ELISA) for the quantification for human factor VII. Thromb Haemost 1986;56:250-5.

38 Poggio M. Specific assays of haemostasis proteins: factor VII. Ric Clin Lab 1990;20(2):139-41.

39 Tietel JM, Bauer KA, Lau HK, et al. Studies on the prothrombin activation pathway utilising radioimmunoassay for the $\mathrm{F} 2 / \mathrm{F} 1+2$ fragment and thrombinantithrombin complex. Blood 1992;59:1086-97.

40 Ceriello A, Giacomello R, Colatutto A, et al. Increased prothrombin fragment $1+2$ in type 1 diabetic patients. Haemostasis 1992;22:50-1.

41 Iwashima Y, Sato T, Watanabe K, et al. Elevation of plasma thrombomodulin level in diabetic patients with early nephropathy. Diabetes 1990;39:983-8.

42 Ford I, Singh TP, Kitchen S, et al. Activation of coagulation in diabetes mellitus in relation to the presence of vascular complications. Diabetic Med 1991;8:322-9.

43 Ceriello A, Giugliano D, Quatraro A, et al. Blood glucose may condition factor VII levels in diabetic and normal subjects. Diabetologia 1988;31:889-91.

4 Kario K, Sakara T, Matsuo T, et al. Factor VII in non-insulin-dependent diabetic patients with microalbuminuria. Lancet 1993;342:1552 (letter)

45 Gruden G, Cavallo-Perin P, Bazzan M, et al. PAI-1 and factor VII activity are higher in IDDM patients with microalbuminuria. Diabetes 1994;43:426-9.

46 Bauer KA, Rosenberg RD. The pathophysiology of the prethrombotic state in humans: insight gained from studies using markers of haemostatic system activation. Blood 1987;70:343-50.

47 Miller GJ, Wilkes HC, Meade TW, et al. Haemostatic changes that constitute the hypercoagulable state. Lancet 1991;338:1079 (letter).

48 Esmon CT. The regulation of natural anticoagulant pathways. Science 1987;235:1348-52.

pathways. Science 1987;235:1348-52.
49 Vingolo EM, De Mattia G, Giusti C, et al. Treatment of nonproliferative diabetic retinopathy with defibrotide in noninsulin-dependent diabetes mellitus: a pilot study. Acta Ophthalmol Scand 1999;77:315-20. 